\title{
A New Approach to Objective Evaluation of Human Visual Acuity
}

\author{
Arkadiusz Hulewicz* \\ Institute of Electrical Engineering and Electronics, Division of Metrology and Optoelectronics, \\ Poznan University of Technology, Piotrowo 3A, 60-965 Poznan
}

Received December 05, 2014; accepted December 18, 2014; published December 31, 2014

\begin{abstract}
The subject matter of this paper refers to objective visual acuity assessment methods using non-invasive measurements of visual evoked potentials (VEP) by means of special electrodes. An attempt was made to classify acquired data, enabling prompt and objective visual acuity evaluation. The purpose of the comparative measurements presented in the paper was to calibrate the peak-to-peak VEP curve sections in a visual acuity function.
\end{abstract}

Visual acuity (VA) refers to the quality of a given person's sight. It is typically expressed as a ratio which enables ophthalmologists to compare a person's vision to that of average people. Visual acuity assessment is traditionally carried out by means of ophthalmological tests using special charts and dependencies taking place between the values of particular points defined for the curves obtained during electrophysiological tests.

The subjective measurement of visual acuity is generally a routine. VA assessment is clearly defined (the norm EN ISO 8596). The measure VA is a numerical parameter determining the extent of vision impairment determined as digits within the range from 0.1 to 1.0. Standard measurement is made on Snellen's charts (the name derived from the author's name - Hermann Snellen, a Dutch ophthalmologist (1834-1908)). The charts are furnished with the so-called optotypes (e.g. letters, digits) of a defined size. According to Snellen's record, VA is given by the dependency: $\mathrm{VA}=\mathrm{D}^{\prime} / \mathrm{D}$, of which $\mathrm{D}^{\prime}$ is the test distance, and $\mathrm{D}$ is the distance from which an "average" eye sees the optotypes on a certain line of the chart. The size of an optotype to be seen without difficulty from such a distance is defined as 1.0. The number of letters increases while the size decreases. The biggest letter on the chart represents an acuity of 0.1 . In a metric record $\mathrm{VA}=6 / 6=1.0$ means that the eye tested is able to read a letter of a defined size from a $6 \mathrm{~m}$ distance and this is $100 \%$ visual efficiency. $\mathrm{VA}<1$ always means weakened visual efficiency.

This test is simple, but its major disadvantage is its totally subjective nature and of key importance is the ability of working with a patient. Patient compliance with examining instructions is very important. This refers

\footnotetext{
*E-mail: arkadiusz.hulewicz@put.poznan.pl
}

particularly to the tests made on children, mentally disabled people and persons suspected of simulation or malingering. Therefore, the possibility of carrying out objective tests is of crucial importance in ophthalmology.

By definition, the visually evoked potential is the averaged encephalographic response, evoked by a repeated visual stimulus recorded with scalp electrodes [1-4]. A standard VEP signal is the averaged response from the whole visual field and optical nerve, evaluating the visual nervous system from the eye to the brain. The VEP curve enables the assessment of retino-cortical conduction in the visual nerve and the visual duct.

In the tests reported in this paper, RETIscan system manufactured by Roland Consult, Germany [7], was applied as a device enabling the acquisition of multifocal electroretinograms as well as visually evoked potentials (Fig. 1). The system is composed of a few connected subassemblies, including two computers: a stimulating computer with a light impulse stimulator and a steering computer which task is to conduct the test and analyze its results. The computer supported RETIsystem allows the users to acquire diagnostic information and analyze it. The set is equipped with specialized RETIsystem software, composed of two sub-programs: RETIscan and RETIport.

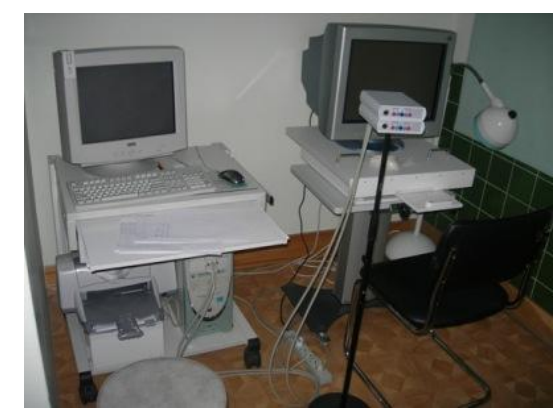

Fig. 1. Illustration of measurements of visual evoked potentials carried out.

Objective visual acuity assessment methods with the use of VEP consist in assessing the changes of electric functional potentials arising in the cerebral cortex area with the use of an appropriate stimulus $[4,5,7,9]$. The 
tests are based on the VEP measurement by means of scalp electrodes. When checking visual acuity, one eye is covered at a time and the vision of each eye is recorded separately, as well as both eyes together. During the SEQSTIMUL test, suggested by the device manufacturer, each eye was stimulated by a series of light stimuli in the form of a black and white chessboard of varying size (Fig. 2). Each measuring cycle consisted of 5 subsequent light stimuli of decreasing squares that made the chessboard. In order to enhance measuring accuracy and minimize interference impact, each test was repeated 100 times for a given chessboard sequence and then the results were averaged. While observing the flashing image generated on the stimulating screen, the patient concentrates on its centre. Being the effect of examined visual path reaction to a light stimulus, the signals allow for topographic assessment of the visual field and are received by means of the three electrodes mentioned above.

The VEP curve and its characteristic parameter are determined for each stimulus size and this is the absolute difference between the maximum and the minimum value of the curve.

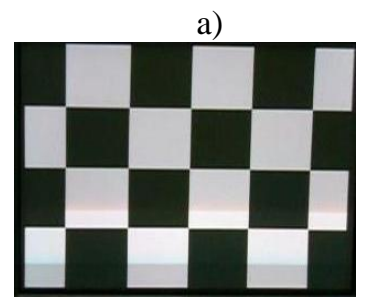

b)

Fig. 2. The SEQ-STIMUL test stimulation source; a) initial stimulus, i.e. 1 of 5 , b) final stimulus, i.e. 5 of 5 .

The averaged VEPs, defined for subsequent stimulus sizes make the specific SEQ-STIMUL curve (Fig. 3); its parameters enabling visual acuity determination. The time of occurrence of the minimum and maximum value of subsequent VEPs is determined for a given curve, as well as the peak-to-peak values "A", being the absolute difference between them. By virtue of the so-determined values it was possible to carry out visual acuity assessment. For the initial stimulation "1", the determined parameter assumes the minimum value "A1" and increases for subsequent stimulations. The size of changes indicates the extent of visual impairment. The more significant the visual impairment, the lower are the changes of subsequent peak-to-peak values [7]. Figures 4 and 5 specify the results of selected comparative tests obtained for a group of 39 people.

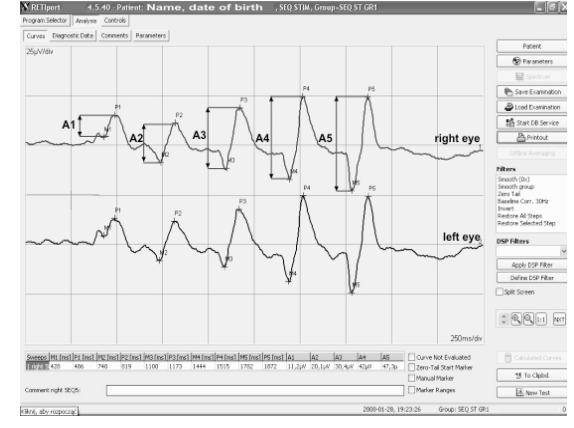

Fig. 3. Examples of the SEQ-STIMUL curves: the peak-to-peak values $\mathrm{A} 1, \mathrm{~A} 2, \mathrm{~A} 3, \mathrm{~A} 4$, and $\mathrm{A} 5$ occur for the subsequent light stimuli 1, $2,3,4$ and 5 , respectively.

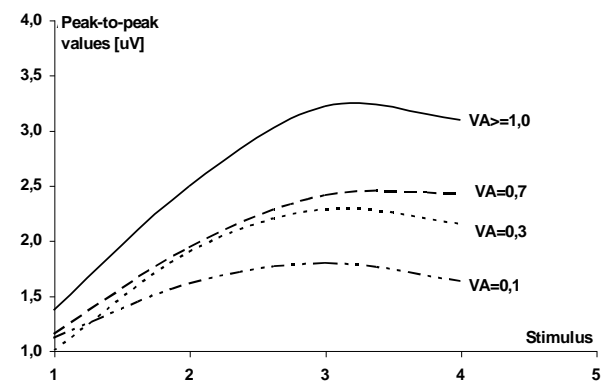

Fig. 4. Calibrating curves of SEQ-STIMUL for different values of visual acuity.

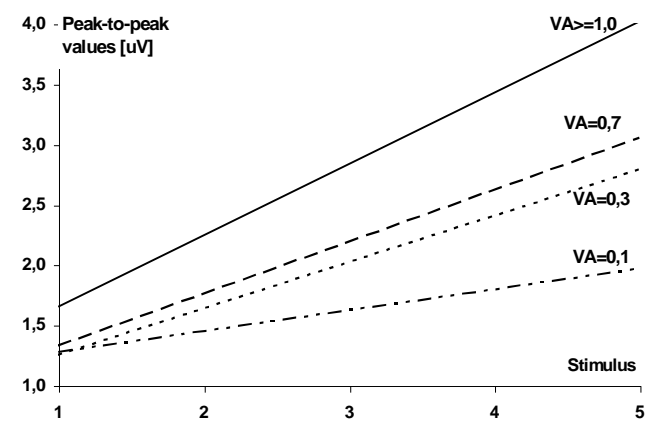

Fig. 5. Calibrating, approximated curves of SEQ-STIMUL for different values of visual acuity.

Each of the persons had his/her visual acuity measured before by means of Snellen's chart. The VA value was correct (i.e. above or equal to 1 ) for 30 people but in the other cases, the visual acuity was impaired to various extents $(\mathrm{VA}<1)$. The A1-A5 values of the specific VEPs were determined for each SEQ-STIMUL test. The extent of the line tendency allows an objective estimation of the VA values. The evaluation of uncertainty for an examination series has allowed the authors to find measurable relation of "A" values to VA values. The value A5 is always largest for $\mathrm{VA}=1.0$. If visual acuity is impaired, the value A5 will decrease in accordance with the extent of weakened sharpness of vision. The specific principles and conditions of measurement are included in the recommendations of the International Society for 
Clinical Electrophysiology of Vision (ISCEV) [6]. The essential difficulty is the lack of standardized intervals determining the correct values of amplitudes and latencies in defined areas of the visual path tested. ISCEV recommends that each laboratory has to establish or confirm its own norms using its own stimuli, recording equipment and parameters. The tests on a selected group of people were made at the Vision Electrodiagnostics Lab with the use of non-invasive measuring gold electrodes and the RETIsystem specialized device and software.

The SEQ-STIMUL signals acquired and then processed were displayed in numerical and graphic forms. The test described by the SEQ-STIMUL device manufacturer has no standardized values enabling fast and objective visual acuity assessment. An attempt was made by the authors to determine such values. For this purpose, a series of tests was carried out in healthy persons and patients with diagnosed visual impairment.

\section{References}

[1] A. Cysewska-Sobusiak, A. Hulewicz, A. Grzybowski, M. Boltrukiewicz, Proc. of the 3rd European Medical and Biological Engineering Conference EMBEC 11, 1-6 (2005).

[2] A. Cysewska-Sobusiak, A. Hulewicz, A. Grzybowski, M. Boltrukiewicz, Proc. of the IEEE International Conference on Virtual Environments, Human-Computer Interfaces, and Measurement Systems VECIMS, 149-153 (2006).

[3] A. Cysewska-Sobusiak, A. Hulewicz, A. Grzybowski, Proc. of the 4th IET International Conference on Advances in Medical, Signal and Information Processing MEDSIP (2008).

[4] J. Heckenlively, G.B. Arden, Principles and practice of clinical electrophysiology of vision (Mosby Year Book, 1991).

[5] M.F. Marmor, A.B. Fulton, G.E. Holder et al., Documenta Ophthalmologica 118, 69-77 (2009)

[6] J.V. Odom, M. Bach, M. Brigell et al., Documenta Ophthalmologica 120, 111-119 (2010)

[7] RETIport21: VEP, EOG, ERG Version 06.02, Instruction manual (Roland Consult, Germany, 2006).

[8] G. Smith, D.A. Atchison, The eye and visual optical instrument (Cambridge University Press, New York, 1997).

[9] W.H. Ridder III, Documenta Ophthalmologica 109, 239-247 (2004). 